\title{
Hiccups, Hemolysis, Elevated Liver Enzymes and Low Plate- lets Syndrome and Hepatic Hematoma
}

\author{
Anna Torgeson ${ }^{\mathrm{a}}$, Nathaniel S Rial ${ }^{\mathrm{b}}$, Richard Temple ${ }^{\mathrm{a}, \mathrm{c}}$
}

\begin{abstract}
Hemolysis, elevated liver enzymes and low platelets (HELLP) syndrome, which affects $0.2-0.9 \%$ of pregnancies, is one of the most difficult obstetric complications to manage. Affected patients can decompensate acutely, and there are a myriad of possible etiologies to evaluate and treat. Serious complications of HELLP syndrome are disseminated intravascular coagulopathy, abruptio placentae and acute renal failure, which are often interdependent. We describe a rare complication of HELLP that manifested itself in an unusual presentation: right shoulder pain, right upper quadrant pain and hiccups which progressed to fetal intolerance of labor due to an acute expansion of a large liver hematoma. This case represents a unique manifestation of the syndrome that provokes further questions in the evaluation and management of patients affected by HELLP syndrome.
\end{abstract}

Keywords: Hemolysis, elevated liver enzymes and low platelets; Cesarean section; Right upper quadrant; Hiccups; Hepatic hematoma

\section{Introduction}

The syndrome of hemolysis, elevated liver enzymes and low platelets (HELLP) affects $0.2-0.9 \%$ of pregnancies overall and $10-20 \%$ of pregnancies complicated by severe pre-eclampsia [1-3]. While thought to be a variant of severe preeclampsia, it can occur without concurrent hypertension in

\footnotetext{
Manuscript accepted for publication March 3, 2014

${ }^{a}$ Family Medicine Residency, Naval Hospital Camp Lejeune, 100 Brewster Blvd, Camp Lejeune, North Carolina 28547, USA

${ }^{\mathrm{b}}$ Internal Medicine Department, Naval Hospital Camp Lejeune, 100

Brewster Blvd, Camp Lejeune, North Carolina 28547, USA

${ }^{\mathrm{c}}$ Corresponding author: Richard Temple, Department of Family Medicine, Naval Hospital Camp Lejeune, Camp Lejeune, NC 28547, USA. Email: Richard.temple@med.navy.mil

doi: http://dx.doi.org/10.14740/jmc1285w
}

$12-18 \%$ of patients [2]. HELLP syndrome often presents with symptoms of right upper quadrant (RUQ) pain, nausea with vomiting, headache or a vague viral-like syndrome [2]. The diagnostic criteria for HELLP syndrome are based on laboratory data (Table 1) [2,3].

Management entails blood pressure control, corticosteroids to promote fetal lung maturity if $<34$ weeks gestation, seizure prophylaxis with magnesium sulfate and preparation for delivery of the fetus. The ultimate treatment for HELLP syndrome is delivery of the fetus, though the method and timing of delivery is dependent on multiple variables including gestational age, stability of the mother, fetal status and favorability of the cervix.

\section{Case Report}

A 29-year-old female, G1P0 at $37+2$ weeks estimated gestational age presented to our labor and delivery triage with a complaint of a 2-week history of steadily worsening upper back, right shoulder and epigastric pain worsened by inspiration and associated with frequent hiccups. She denied headache or scotoma. Her blood pressures ranged 133-173/86-98 $\mathrm{mmHg}$, and her exam was significant for mild tenderness of her RUQ. Laboratory data revealed: trace protein on urinalysis, platelets of $76,000 / \mu \mathrm{L}$ (normal $179,000-408,000 / \mu \mathrm{L}$ ), hemoglobin $12.3 \mathrm{~g} / \mathrm{dL}$ (normal 10.9 - $14.3 \mathrm{~g} / \mathrm{dL}$ ), aspartate aminotransferase of $282 \mathrm{U} / \mathrm{L}$ (normal 15 - $37 \mathrm{U} / \mathrm{L}$ ), alanine aminotransferase of $374 \mathrm{U} / \mathrm{L}$ (normal $12-78 \mathrm{U} / \mathrm{L}$ ) and lactate dehydrogenase of $444 \mathrm{U} / \mathrm{L}$ (normal 84 - $246 \mathrm{U} / \mathrm{L}$ ). Fetal heart rate tracing revealed a baseline of 150 beats per minute, moderate variability, presence of accelerations and absence of decelerations. The patient was diagnosed with HELLP syndrome, admitted to labor and delivery, consented for an induction of labor, and begun on pitocin (Bishop score of 9), as well as magnesium sulfate for seizure prophylaxis.

The patient progressed to $4 \mathrm{~cm}$ of cervical dilation at which time she experienced acute worsening of her RUQ pain, shortness of breath, tachypnea and tachycardia. The fetal heart rate tracing revealed a loss of variability as well as a late deceleration into the 70 beats per minute which did not respond to intrauterine resuscitative measures. The patient 
Table 1. Diagnostic Criteria for HELLP Syndrome [2, 3]

Tennessee classification

\begin{tabular}{ll}
\hline Platelets & $\leq 100 \times 10^{9} / \mathrm{L}$ \\
AST & $\geq 70 \mathrm{U} / \mathrm{L}$ \\
LDH & $\geq 600 \mathrm{U} / \mathrm{L}$ \\
\hline
\end{tabular}

Additional indicators of hemolysis: elevated indirect serum bilirubin, low serum haptoglobin and drop in hemoglobin levels. HELLP: hemolysis, elevated liver enzymes and low platelets; AST: aspartate aminotransferases; LDH: lactate dehydrogenase.

was taken to the operating room for delivery via an emergent cesarean section under general anesthesia. Pre-operative hemoglobin was $8.0 \mathrm{~g} / \mathrm{dL}$ and platelet count was $43,000 / \mu \mathrm{L}$. The patient's blood loss during the operation was approximately $1,000 \mathrm{~mL}$.

Post-operatively the patient became progressively tachycardic (140 - 150 beats per minute) with inadequate urine output. She received a transfusion of four units of packed red blood cells, one unit of platelets, two units of fresh frozen plasma in addition to $4 \mathrm{~L}$ of lactated ringers. Magnesium sulfate was continued.

While her clinical symptoms improved with the above resuscitative measures her international normalized ratio as well as liver associated enzymes continued to rise so she was transferred to a tertiary care hospital (Table 2). Upon arrival at the receiving hospital an abdominal computed tomography (CT) scan was obtained (Fig. 1, 2), which demonstrated a large hepatic hematoma that was surrounded by the capsule of the liver. The estimated blood volume was 2 - 3 L. The patient was managed conservatively with serial imaging and hemodynamic monitoring. She did not require further surgical intervention. After 1 week of monitoring, she was discharged to outpatient follow-up.

\section{Discussion}

In a prospective cohort of 442 pregnancies complicated by HELLP syndrome, Sibai noted the following rates of complications: disseminated intravascular coagulopathy (21\%), abruptio placentae $(16 \%)$, acute renal failure $(8 \%)$, severe ascites $(8 \%)$, pulmonary edema $(6 \%)$, pulmonary effusions $(6 \%)$ and maternal death (1\%). Additionally, subcapsular hepatic hematoma occurred in four patients $(0.9 \%)$. In these four patients, three of the fetuses died in utero and one mother died as a result of a rupture of the hepatic hematoma [4].

Barton reports a series of 34 patients with HELLP syndrome and severe RUQ abdominal pain in association with shoulder pain, neck pain or relapsing hypotension, 13 of whom were found to have a subcapsular hepatic hematoma,

Table 2. Laboratory Data

\begin{tabular}{lllllllll}
\hline Time & Date & AST & ALT & LDH & Bilirubin & Creatinine & Hgb/Hct & Platelets \\
\hline 0800 & $11-$ Sep-12 & - & - & - & - & - & $11.4 / 31.7$ & 250 \\
0300 & $13-$ Nov-12 & 282 & 374 & 444 & 0.4 & 0.40 & $12.3 / 35.1$ & 76 \\
1015 & $13-$ Nov-12 & - & - & - & - & - & $8.0 / 23.4$ & 43 \\
1250 & $13-N o v-12$ & 897 & 726 & 939 & 1.10 & 1.0 & $8.1 / 24.4$ & 50 \\
\hline
\end{tabular}

AST: aspartate aminotransferases; ALT: alanine aminotransferases; LDH: lactate dehydrogenase; Hgb: hemoglobin; Hct: hematocrit. 


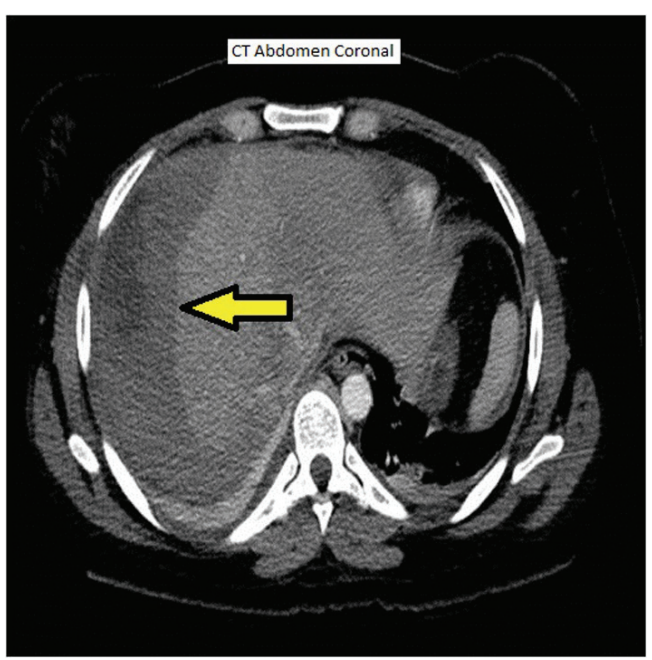

Figure 1. CT coronal view abdomen.

six were found to have intraparenchymal hemorrhage and four experienced a rupture of the hematoma. Overall rates of complications in the 34 patients were: disseminated intravascular coagulopathy $(53 \%)$, acute renal failure $(41 \%)$, severe ascites $(62 \%)$, pulmonary edema (26\%) and pulmonary effusions $(53 \%)$. Additionally, there were nine perinatal deaths and one maternal death [5].

Hepatic hematoma formation is thought to be due to fibrin deposition in hepatic arterioles and sinusoids resulting in periportal necrosis and hepatic hemorrhage contained within Glisson's capsule [5-7]. Rupture of the hepatic hematoma can result in maternal mortality in up to $86 \%$ of cases [3]. Transabdominal ultrasound, CT and magnetic resonance imaging can all be utilized to detect, measure and monitor subcapsular hepatic hematomas [8].

This is a unique case of a pregnant patient presenting with HELLP syndrome and symptoms of subdiaphragmatic

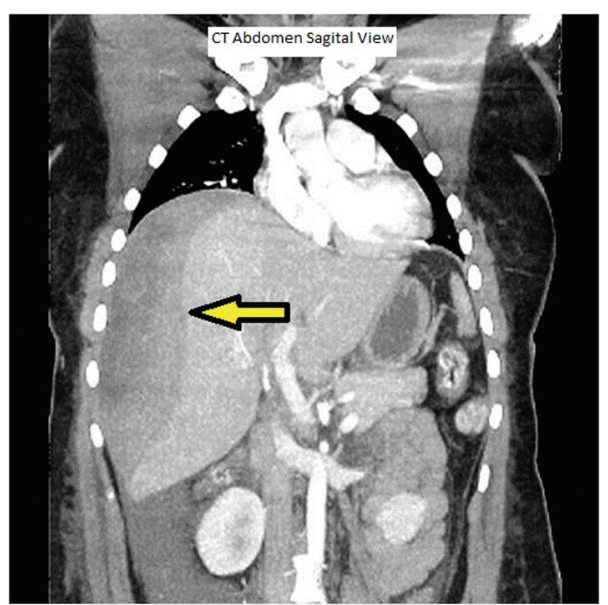

Figure 2. CT sagital view abdomen. irritation: right shoulder, RUQ and epigastric pain with the addition of hiccups. Subdiaphragmatic irritations to include hepatoma, liver metastasis and subdiaphragmatic abscesses are known causes of hiccups [9]. The referred pain as well as the hiccups was likely the result of phrenic nerve irritation due to an underlying hepatic hematoma. To our knowledge, this is the only published case report of a patient presenting with hiccups as a part of her constellation of symptoms of HELLP syndrome. During her induction of labor, we suspect she experienced an acute expansion of this hematoma resulting in decompensation in fetal status, worsening RUQ pain and shortness of breath. Other possible etiologies of these acute symptoms in this clinical setting include pulmonary embolus, amniotic fluid embolus, placental abruption and uterine rupture.

A high index of suspicion for and early recognition of a pre-existing hepatic hematoma in a patient with HELLP syndrome can result in expediting delivery of the fetus as well as prompt transfer to a tertiary care center where subspecialty services are available to manage potential complications. It is our recommendation to obtain hepatic imaging whenever there is evidence of diaphragmatic irritation (referred right shoulder pain, RUQ pain, epigastric pain and hiccups) in a patient with presenting HELLP syndrome.

\section{Conflicts of Interest}

The authors have no conflicts of interest to declare.

\section{References}

1. Abildgaard U, Heimdal K. Pathogenesis of the syndrome of hemolysis, elevated liver enzymes, and low platelet count (HELLP): a review. Eur J Obstet Gynecol Reprod Biol. 2013;166(2):117-123.

2. Sibai BM. Diagnosis, controversies, and management of the syndrome of hemolysis, elevated liver enzymes, and low platelet count. Obstet Gynecol. 2004;103(5 Pt 1):981-991.

3. Haram K, Svendsen E, Abildgaard U. The HELLP syndrome: clinical issues and management. A Review. BMC Pregnancy Childbirth. 2009;9:8.

4. Sibai BM, Ramadan MK, Usta I, Salama M, Mercer BM, Friedman SA. Maternal morbidity and mortality in 442 pregnancies with hemolysis, elevated liver enzymes, and low platelets (HELLP syndrome). Am J Obstet Gynecol. 1993;169(4):1000-1006.

5. Barton JR, Sibai BM. Hepatic imaging in HELLP syndrome (hemolysis, elevated liver enzymes, and low platelet count). Am J Obstet Gynecol. 1996;174(6):18201825; discussion 1825-1827.

6. Aarnoudse JG, Houthoff HJ, Weits J, Vellenga E, Huis- 
jes HJ. A syndrome of liver damage and intravascular coagulation in the last trimester of normotensive pregnancy. A clinical and histopathological study. Br J Obstet Gynaecol. 1986;93(2):145-155.

7. Xavier P, Melo R, Amandio V, Beires J, Pereira-Leite L. Subcapsular hepatic hematoma in an otherwise uncomplicated pregnancy. Arch Gynecol Obstet. 2002;266(1):44-45.
8. Wicke C, Pereira PL, Neeser E, Flesch I, Rodegerdts EA, Becker HD. Subcapsular liver hematoma in HELLP syndrome: Evaluation of diagnostic and therapeutic options--a unicenter study. Am J Obstet Gynecol. 2004;190(1):106-112.

9. Marinella MA. Diagnosis and management of hiccups in the patient with advanced cancer. J Support Oncol. 2009;7(4):122-127, 130. 